\title{
Percepción de barreras para la práctica del ejercicio físico en adolescentes
}

\author{
gallegos
}

\section{Perception of barriers that hinder the practice of physical exercise in Galician}

\section{adolescents}

\author{
Iago Portela Pino*, Antonio López Castedo* \\ *Universidade de Vigo,
}

\begin{abstract}
Resumen
El presente trabajo tiene como objetivo identificar las barreras que dificultan la práctica del ejercicio físico en la adolescencia. Para ello, se llevó a cabo un estudio descriptivo y transversal con 342 estudiantes de Educación Secundaria Obligatoria y Bachillerato $(\mathrm{M}=15.02 \pm 1.86)$, a través del Autoinforme de Barreras para la Práctica del Ejercicio Físico (ABPEF). Los resultados obtenidos muestran como principales barreras que dificultan la práctica del ejercicio físico, las obligaciones o falta de tiempo y la fatiga o pereza. Por el contrario, las barreras con menor peso serían la imagen corporal o ansiedad física-social y el ambiente o las instalaciones.
\end{abstract}

Palabras clave: [ejercicio físico, barreras, adolescencia, ABPEF]

\begin{abstract}
This paper aims to identify the barriers that hinder the practice of physical exercise in adolescence. To do so, was conducted a descriptive and cross-sectional study with 342 students of compulsory secondary education and Bachillerato $(M=15.02 \pm 1.86)$, through the self-report of barriers for the practice of physical exercise (ABPEF). The results show as main barriers that hinder the practice of physical exercise, obligations or lack of time and fatigue or laziness. On the other hand, barriers with less weight would be fisica-social anxiety, body image or facilities and the environment.
\end{abstract}

Keywords: [physical exercise, barriers, adolescence, ABPEF]

En la actualidad se manifiesta una tendencia al reconocimiento de los beneficios que aporta la práctica regular del ejercicio físico a la salud y a la calidad de vida. La evidencia científica señala que el movimiento corporal es un factor esencial de la actividad física para contrarrestar el sedentarismo (American College of Sports Medicine, 2000). No obstante, se ha constatado un descenso en la práctica del ejercicio físico conforme los jóvenes avanzan en edad, siendo la etapa adolescente donde se produce un mayor abandono (Cairney, Veldhuizen, Kwan, Hay, \& Faught, 2014; Currie et al., 2008). En consecuencia, el análisis de las barreras que dificultan la práctica del ejercicio físico es muy importante no solo para poder evitarlas sino también porque la percepción de barreras se asocia con una mayor prevalencia de inactividad física en el tiempo libre de los adolescentes (Dias, Loch \& Ronque, 2015).

Por otro lado, se sabe que durante la adolescencia, la actividad física, disminuye con consecuencias potencialmente graves a largo plazo para la salud física y mental (Langguth et al., 2015). Entre las barreras para el ejercicio físico aparecen varios factores, entre los que destacan: falta de tiempo para la práctica (Awadalla et al., 2014), presión de las tareas educativas (Peykari et al. 2015), falta de un entorno seguro para la práctica de la actividad física (Rajaraman et al., 2015), problemas de salud (Nicholson, Khademi y Moghadasian, 2013) y sobre todo, las responsabilidades familiares, el sentimiento de culpa, la falta de apoyo y las limitaciones de programación y trabajo (Mailey, Huberty, Dinkel \& McAuley, 2104).

No obstante en este estudio, al igual que Niñerola, Capdevila y Pinatel (2006), se hará referencia a cuatro tipos de barreras, imagen corporal o ansiedad física social (vergüenza de enseñar el cuerpo en público o miedo a evaluaciones, bromas y críticas sobre nuestro cuerpo), fatiga o pereza (razones intrínsecas de tipo personal como pereza, falta de voluntad o malestar), obligaciones $\mathrm{o}$ falta de tiempo (organización o administración del tiempo debido a cuestiones académicas o sociales) y ambiente o instalaciones (dificultad para acceder a espacios donde se pueda practicar ejercicio físico, deficiencias en las instalaciones).

Por estas razones, nuestro objetivo se centra en conocer las principales barreras que dificultan la práctica regular de ejercicio físico en los adolescentes, lo cual ayudaría a optimizar su inicio o continuación, así como minimizar su abandono.

\section{Método}

\section{Participantes}

La muestra estuvo conformada por 342 adolescentes de la Comunidad Autónoma de Galicia (Tabla 1). Se utilizó como marco poblacional base, los datos y cifras 
de la enseñanza no universitaria (Xunta de Galicia, 2015). De esta manera, la muestra se distribuyó de la siguiente manera: $45.9 \%$ de los adolescentes encuestados son mujeres y $54.1 \%$ son hombres. Además, en referencia a la edad: $10.8 \%$ tiene 12 años, $16.1 \%$ tiene 13 años, $16.1 \%$ tiene 14 años, $8.5 \%$ tiene 15 años, $21.1 \%$ tiene 16 años, $21.6 \%$ tiene 17 años y un $5.8 \%$ tiene 18 años. La media de edad fue de $15.1(D T=$ 1.86).

Tabla 1.

Características de la muestra en función de las variables género y edad

\begin{tabular}{llcc}
\hline & & \multicolumn{2}{c}{ Muestra (342 alumnos) } \\
& & Frecuencias & Porcentajes \\
\hline \multirow{2}{*}{ Género } & Femenino & 157 & $45.9 \%$ \\
& Masculino & 185 & $54.1 \%$ \\
\hline \multirow{6}{*}{ Edad } & 12 años & 37 & $10.8 \%$ \\
& 13 años & 55 & $16.1 \%$ \\
& 14 años & 55 & $16.1 \%$ \\
& 15 años & 29 & $8.5 \%$ \\
& 17 años & 72 & $21.1 \%$ \\
& 18 años & 74 & $21.6 \%$ \\
TOTAL & & 20 & $5.8 \%$ \\
\hline
\end{tabular}

\section{Instrumento}

Para analizar las barreras o dificultades percibidas en relación al ejercicio físico se ha utilizado el Autoinforme de Barreras para la Práctica del Ejercicio Físico (ABPEF; Capdevila, 2005), adaptado por Niñerola, Capdevila y Pintanel (2006). Esta versión consta de 17 ítems que se responden en una escala Likert de 0 (razón poco probable que me impide practicar ejercicio físico en próximas semanas) a 10 (razón muy probable que me impide practicar ejercicio físico) puntos. El estudio original informa de cuatro subescalas diferentes (imagen corporal/ansiedad física y social; fatiga/pereza; obligaciones/falta de tiempo; ambiente/instalaciones) con una buena fiabilidad y adecuada validez.

\section{Procedimiento}

El cuestionario se administró de manera colectiva en estudiantes de educación secundaria obligatoria y bachillerato en horario regular de clases, durante el curso 2015-16. Después de comunicar las instrucciones oportunas y previo consentimiento informado (centro y familias), todo el alumnado cumplimentó de forma voluntaria la información solicitada. Antes de contestar al cuestionario, los estudiantes fueron informados de la finalidad del estudio, solicitándoles su participación voluntaria y anónima. Las instrucciones explicativas fueron las mismas en todas las aulas y por el mismo equipo investigador, a fin de evitar un factor de sesgo. Se cumplieron los protocolos éticos de investigación con especial énfasis en la confidencialidad.

\section{Análisis de los datos}

Se llevó a cabo un estudio exploratorio-descriptivo analizando la frecuencia de cada tipo de barrera hacia la práctica del ejercicio físico percibida por los adolescentes, calculando las medias y porcentajes de respuesta a cada opción de la escala en cada ítem. Los análisis fueron realizados con el paquete estadístico SPSS 21.0 (IBM Corp., 2012).

\section{Resultados}

El nivel percibido para cada tipo e indicador específico de barreras para la práctica del ejercicio físico se muestra en la tabla 1. Atendiendo a estos resultados, se observa que el tipo de barrera en la práctica del ejercicio físico percibido como más habitual por parte del alumnado de ESO y Bachillerato de Galicia es la obligación o falta de tiempo, seguido de la fatiga o pereza. En concreto, destacan impedimentos como estimar que tienen demasiado trabajo, tener vagancia, no contar con tiempo suficiente, falta de voluntad, dolores musculares, y sobre todo, cansancio o fatiga.

En un término medio, se sitúan fundamentalmente las barreras que hacen referencia a imagen corporal y ansiedad físico-social, aduciendo razones como "pensar que la otra gente está en mejor forma que yo", "sentir mi aspecto físico peor que el de los demás" o "pensar que los demás juzgan mi apariencia física”.

Finalmente, entre las barreras menos percibidas como impedimento a la práctica del ejercicio físico se encuentra también la imagen corporal y ansiedad físicosocial, con impedimentos como "sentir incomodidad por el aspecto que tengo con ropa deportiva", "encontrarme a disgusto con la gente que hace ejercicio conmigo" o "sentir vergüenza porque me están mirando mientras hago ejercicio físico"; o el ambiento y las instalaciones con justificaciones del tipo "las instalaciones o los monitores no son adecuados" o "estar demasiado lejos del lugar donde puedo hacer ejercicio físico". En este aspecto es recurrente el pensar que otra gente está más en forma, que su aspecto físico es peor que el de los demás, o sentir incomodidad por su apariencia física. 
Tabla 2.

Descriptivos de los items del ABPEF con el total de la muestra de ESO y Bachiller $(N=342)$, por orden de frecuencia

\begin{tabular}{|c|c|c|c|c|c|c|c|c|c|c|}
\hline \multirow{2}{*}{ Orden } & \multirow{2}{*}{ Ítem } & \multirow{2}{*}{ Factor } & \multirow{2}{*}{ Enunciado } & \multirow{2}{*}{ M } & \multirow{2}{*}{ D.T. } & \multicolumn{4}{|c|}{ Poca Probabilidad (\%) } & \multirow{2}{*}{$\begin{array}{c}\text { Mucha } \\
5\end{array}$} \\
\hline & & & & & & 1 & 2 & 3 & 4 & \\
\hline $1^{\mathrm{o}}$ & 4 & $\mathrm{O} / \mathrm{FT}$ & Tener demasiado trabajo & 2.60 & 1.43 & 33.9 & 14.3 & 24 & 13.2 & 14.6 \\
\hline $2^{\circ}$ & 2 & $\mathrm{~F} / \mathrm{P}$ & Tener pereza & 2.23 & 1.39 & 44.2 & 21.1 & 14.6 & 8.2 & 12 \\
\hline $3^{\circ}$ & 11 & $\mathrm{O} / \mathrm{FT}$ & $\begin{array}{l}\text { No encontrar el tiempo necesario } \\
\text { para el ejercicio }\end{array}$ & 2.22 & 1.39 & 46.5 & 16.1 & 15.8 & 11.7 & 9.9 \\
\hline $4^{\mathrm{o}}$ & 9 & $\mathrm{~F} / \mathrm{P}$ & Faltar de voluntad para ser constante & 1.89 & 1.25 & 57.6 & 14.9 & 14.6 & 6.1 & 6.7 \\
\hline $5^{\circ}$ & 5 & $\mathrm{~F} / \mathrm{P}$ & $\begin{array}{l}\text { Tener "agujetas" o dolores } \\
\text { musculares a consecuencia del } \\
\text { ejercicio }\end{array}$ & 1.69 & 1.06 & 63.2 & 16.4 & 12 & 5.6 & 2.9 \\
\hline $6^{\circ}$ & 7 & $\mathrm{O} / \mathrm{FT}$ & $\begin{array}{l}\text { Tener demasiadas obligaciones } \\
\text { familiares }\end{array}$ & 1.67 & 1.04 & 63.2 & 16.7 & 12.6 & 5 & 2.6 \\
\hline $7^{\circ}$ & 12 & $\mathrm{~F} / \mathrm{P}$ & $\begin{array}{l}\text { Notar cansancio o fatiga de forma } \\
\text { habitual a lo largo del día }\end{array}$ & 1.65 & 1.11 & 67.3 & 14 & 9.6 & 4.4 & 4.7 \\
\hline $8^{\circ}$ & 10 & $\mathrm{IC} / \mathrm{AFS}$ & $\begin{array}{l}\text { Pensar que la otra gente está en } \\
\text { mejor forma que yo }\end{array}$ & 1.63 & 1.17 & 72.2 & 9.1 & 7.9 & 5.3 & 5.6 \\
\hline $9^{\circ}$ & 6 & IC/AFS & $\begin{array}{l}\text { Sentir mi aspecto físico peor que el } \\
\text { de los demás }\end{array}$ & 1.52 & 1.01 & 73.4 & 11.4 & 7.6 & 4.7 & 2.9 \\
\hline $10^{\circ}$ & 13 & $\mathrm{IC} / \mathrm{AFS}$ & $\begin{array}{l}\text { Pensar que los demás juzgan mi } \\
\text { apariencia física. }\end{array}$ & 1.51 & 1.05 & 75.1 & 10.5 & 5.6 & 5 & 3.8 \\
\hline $11^{\circ}$ & 8 & $\mathrm{~F} / \mathrm{P}$ & $\begin{array}{l}\text { No estar "en forma" para practicar } \\
\text { ejercicio }\end{array}$ & 1.51 & .99 & 74 & 9.6 & 9.9 & 3.8 & 2.6 \\
\hline $12^{\circ}$ & 1 & $\mathrm{~F} / \mathrm{P}$ & $\begin{array}{l}\text { Cansarme demasiado durante el } \\
\text { ejercicio o miedo a lesionarme }\end{array}$ & 1.50 & .89 & 69.3 & 17.5 & 8.5 & 2.9 & 1.8 \\
\hline $13^{\circ}$ & 14 & $\mathrm{~A} / \mathrm{I}$ & $\begin{array}{l}\text { Estar demasiado lejos del lugar } \\
\text { donde puedo hacer ejercicio }\end{array}$ & 1.48 & .88 & 71.3 & 15.2 & 8.5 & 3.8 & 1.2 \\
\hline $14^{\circ}$ & 16 & IC/AFS & $\begin{array}{l}\text { Sentir vergüenza porque me están } \\
\text { mirando mientras hago ejercicio }\end{array}$ & 1.45 & 1.01 & 78.9 & 8.5 & 5.3 & 3.2 & 4.1 \\
\hline $15^{\circ}$ & 15 & IC/AFS & $\begin{array}{l}\text { Encontrarme a disgusto con la gente } \\
\text { que hace ejercicio conmigo }\end{array}$ & 1.34 & .80 & 79.5 & 11.7 & 5.3 & 1.8 & 1.8 \\
\hline $16^{\circ}$ & 17 & $\mathrm{~A} / \mathrm{I}$ & $\begin{array}{l}\text { Que las instalaciones o los monitores } \\
\text { no sean adecuados }\end{array}$ & 1.33 & .81 & 80.1 & 12.3 & 3.8 & 1.8 & 2 \\
\hline $17^{\circ}$ & 3 & IC/AFS & $\begin{array}{l}\text { Sentir incomodidad por el aspecto } \\
\text { que tengo con ropa deportiva }\end{array}$ & 1.31 & .83 & 83.9 & 7.3 & 4.1 & 2.6 & 2 \\
\hline
\end{tabular}

\section{Discusión}

A pesar del reconocimiento de los enormes beneficios y calidad de vida que aporta la práctica regular de ejercicio físico, dos hechos constatables empíricamente marcan el período adolescente, un descenso considerable en los niveles de actividad físico-deportiva (Román et al., 2008) y una mayor tasa de abandono (Currié et al., 2008). Por esta razón, el presente estudio ha analizado las principales barreras que dificultan la práctica del ejercicio físico en el periodo adolescente, según la opinión del alumnado de Educación Secundaria Obligatoria y Bachillerato, aportando información relevante para el autodiagnóstico de los centros de enseñanza en este campo. El objetivo consiste en conocer las principales razones que llevan a los adolescentes al sedentarismo.

Así pues, en primer lugar, se ha puesto de manifiesto que la principal barrera que impide o dificulta la práctica del ejercicio físico en los adolescentes son las obligaciones o falta de tiempo, seguida de la fatiga o pereza. Dichos resultados coinciden con los estudios realizados por Gómez, Ruiz, García, Flores y Barbero (2008), Martínez, Gómez, Abraldes y Rodríguez (2011) y Serra, Generelo y Zaragoza (2010), al señalar como principales barreras que llevan al abandono de la práctica del ejercicio físico la falta de tiempo, la pereza, la desgana personal, la incompatibilidad de horarios, y el sentirse cansado a causa del trabajo o estudio.

Por el contrario, entre las barreras de menor influencia en la práctica de ejercicio físico en este periodo serían la imagen corporal o ansiedad físico-social y el ambiente o las instalaciones. También Granero, Gómez, Abraldes y Rodríguez (2011) afirman que la apariencia es la razón menos valorada en la práctica del ejercicio físico.

En conclusión, en importante reducir los índices de abandono y aumentar los beneficios incorporados a la práctica físico-deportiva continuada (Martínez et al., 2012) a través de estrategias de intervención más cercanas (personalizadas), multimodales y centradas en individuos modificables, entornos familiares y sobre todo en los contextos ambientales (Huang et al., 2008). 


\section{Referencias bibliográficas}

American College of Sports Medicine (2000). ACSM'S guidelines for exercise testing and prescription (6th ed.). Philadelphia, PA: Lippincott, Williams, y Wilkins.

Awadalla, N.J., Aboelyazed, A.E., Hassanein, M.A., Khalil, S.N., Aftab, R. Gaballa I.I., \& Mahfouz, A.A. (2014). Assessment of physical in activity and perceived barriers to physical activity among health college students, south-western Saudi Arabia, EMHJ, 20(10), 596-604.

Cairney, J., Veldhuizen, S., Kwan, M., Hay, J., \& Faught, B. E. (2014). Biological Age and Sex-Related Declines in Physical Activity during Adolescence. Medicine and Science in Sports and Exercise, 46(4), 730-735.

Capdevila, L. (2005). Actividad fisica y Salud. Barcelona, Espana: Miracle.

Huang, B., Gillman, M.W., Field, A.E., Austin, S.B., Colditz, G.A., \& Frazier, A.L. (2008). Patterns and Determinants of Physical Activity in U.S. Adolescents. Journal of Adolescent Health, 42(4), 369-377.

Langguth N., Könen T., Matulis, S., Steil R., Gawrilow, C., \& Stadler, G. (2015). Barriers to Physical Activity in Adolescents. A Multidimensional Approach. Zeitschrift für Gesundheitspsychologie, 23, 47-59. doi: 10.1026/0943-8149/a000136.

Mailey, E.L., Huberty, J., Dinkel, D., \& McAuley, E. (2104). Physical activity barriers and facilitators among working mothers and fathers. BMC Public Health, 14(657), 1-9. doi: 10.1186/1471-2458-14-657

Martínez, A. C., Chillón, P., Martín-Matillas, M., Pérez, I., Castillo, R., Zapatera, B., ...\& Delgado-Fernández, M. (2012). Actitudes hacia la práctica de actividad físico-deportiva orientada a la salud en adolescentes españoles: Estudio AVENA. Retos. Nuevas tendencias en Educación Física, Deporte $y$ Recreación, 22, 43-48.

Nicholson, T., Khademi, H., \& Moghdasian, M.H. (2013). The role of marine $n-3$ fatty acids in improving cardiovascular health: a review. Food \& Function, 4(3), 357-365.

Niñerola, J., Capedvila, L., \& Pintanel, M. (2006). Barreras percibidas y actividad física: el autoinforme de barreras para la práctica de ejercicio físico. Revista de Psicología del Deporte, 15(1), 53-69.

Peykari N., Eftekhari M.B., Tehrani F.R., Afzali H.M., Hejazi F., Atoofi M., Qorbani M., Asayesh H., \& Djalalinia S. (2015). Promoting physical activity participation among adolescents: The barriers and the suggestions. International Journal of Preventive Medicine, 6:12. doi: 10.4103/2008-7802.151820.

Rajaraman D., Correa N., Punthakee Z., Lear S.A., Jayachitra, M., \& Swaminathan S. (2015). Perceived Benefits, Facilitators, Disadvantages, and Barriers for Physical Activity Amongst South Asian Adolescents in India and Canada. Journal of Physical Activity \& Health, 12(7), 931-941.
Currie C., Gabhainn S., Godeau E., Roberts C., Smith R., ... Currie, D. (2008). Inequalities in young people's health. HBSC international report from the 2005/2006 survey. Copenhagen: World Health Organization.

Dias D.F., Loch, M.R., \& Ronque E.R.V. (2015). Perceived barriers to leisure-time Physical activity and associated factors in adolescents. Ciencia \& Saude Coletiva, 20(11), 3339-3350.

Gómez, M., Ruiz J.F., García, E., Flores, G., \& Barbero, G. (2008). Razones que influyen en la inactividad físico-deportiva en la Educación Secundaria Post Obligatoria. Retos. Nuevas Tendencias en Educación Física, Deporte y Recreación, 14, 80-85.

Granero, A., Gómez, M., Abraldes, J.A., \& Rodríguez, N. (2011). Motivos de práctica en el ámbito de la actividad física no competitiva. Espiral. Cuadernos del Profesorado, 4(7), 15-22.

Roman, B., Serra-Majem, L., Ribas-Barba, L., PerezRodrigo, C., \& Aranceta, J. (2008). How many children and adolescents in Spain comply with the recommendations on physical activity? Journal of Sports Medicine and Physical Fitness, 48(3), 380387.

Serra, J.R, Generelo, E., \& Zaragoza, J. (2010). Barreras para la realización de actividad física en adolescentes de la provincia de Huesca. Revista Internacional de Medicina y Ciencias de la Actividad Física y El Deporte, 10(39), 470-482. 